\title{
PHOTOCHEMICAL PHASE TRANSITION OF POLYMER LIQUID CRYSTALS
}

\author{
TOMIKI IKEDA, TAKEO SASAKI and KUNIHIRO ICHIMURA \\ Division of Photochemical Process, \\ Research Laboratory of Resources Utilization, \\ Tokyo Institute of Technology, \\ 4259 Nagatsuta, Midori-ku, Yokohama 227, JAPAN
}

\begin{abstract}
Photochemical phase transition of polymer liquid crystals (PLCs) was explored for PLCs with mesogenic side-chain phenylbenzoate and cyanobiphenyl. The photochemical phase transition was triggered by photochemical reaction of photoresponsive molecules incorporated physically or covalently into PLCs as a result of lowering of phase transition temperature of the mixtures below the irradiation temperature. Factors affecting the photochemical phase transition are discussed and resolution of stored image was examined.
\end{abstract}

\section{Introduction}

Studies on the photon-mode response of LCs started in the late 1960s when Haas et al. reported a change in selective reflectivity of a cholesteric LC mixture composed of cholesteryl bromide and other cholesteryl derivatives on photoirradiation [1]. They also found that irradiation with a UV light of LC cells containing mesogens with stilbene units in the molecules brought about trans - cis isomerization of the stilbene units, causing simultaneously N - I phase transition of the host LC. This was interpreted as a result of lowering of the $\mathrm{N}$ I phase transition temperature (Tni) of the mixture [2]. Since then, many studies on the photoresponsiveness of LCs have been reported [3].

Advantage of the photon-mode recording over the heat-mode recording lies in superior resolution and possibility of multiplex recording in the photon-mode recording [4]. Diffusion of heat evidently worsens the resolution. Variety of information associated with the photon-mode recording (energy, polarization and coherency) are also advantages of the photon-mode recording [4]. For a high sensitivity and a high signal to noise ratio $(\mathrm{S} / \mathrm{N})$ of the recording, other methods 
than direct reading of the photochemical reaction are to be developed. Vision is a good model, in which a photochemical reaction occurring at a local site of visual cells causes conformational change of rhodopsin, leading to amplification of the photosignals [5].

Much attention has recently been paid to polymer liquid crystals (PLCs) as optical image storage materials in view of a phenomenon of glass transition and processability of the polymers [6]. Below the glass transition temperature (Tg), the segmental motion of the polymer chains is frozen-in, thereby the stored information can be kept stable for a long period. Furthermore, cell-free polymer films with the ability of long-term storage are evidently favored from a practical point of view.

Shibaev et al. [7] and Coles and Simon [8] reported the laser-addressed PLC storage devices in which heat-mode recording was exclusively employed. Photon-mode image storage in the PLCs was first demonstrated by Eich and Wendorff as "holographic" optical storage, in which photoirradiation caused isomerization of the photochromic groups incorporated into the PLCs, thus inducing "grating" in the PLCs [9]. We have demonstrated photochemically induced isothermal phase transition in liquid crystals aiming at optical image storage systems [10,11]. The working principle of our systems is isothermal phase transition of the liquid crystals at the irradiation site; thus, we can expect high contrast and high signal-to-noise ratio for the stored information. In this paper, the photochemical phase transition behaviors of PLCs with side-chain mesogenic moieties are described, focusing factors to affect the photochemical phase transition in order to construct effective optical image storage systems.

\section{Experimentals}

\section{Materials}

Structure of PLCs used in this study is shown in Fig. 1. Acrylates with mesogenic phenylbenzoate (APBn) and cyanobiphenyl (ACBn) in the side chain and acrylates with side-chain azobenzene moieties (AAZOm) were prepared by the methods already reported [11]. 4-Butyl-4'-methoxyazobenzene (BMAB) was prepared and purified as reported previously [10]. Polymerization was conducted in tetrahydrofuran (THF) or benzene by the use of AIBN as an initiator. In copolymerization, conversion was always kept low (< $15 \%)$. All polymers were purified by repeated precipitation from chloroform solution into methanol.

\section{Characterization of Polymers}

Molecular weight (MW) of the polymers was determined by gel permeation 
chromatography (GPC; eluent, chloroform) calibrated with standard polystyrenes. GPC was also employed for fractionation of the polymers. Liquid crystalline behavior and phase transition behavior were examined on an Olympus model BHSP polarizing microscope equipped with a Mettler hot stage model FP-80 and FP-82. Thermodynamic properties were determined with a differential scanning calorimeter (DSC; SEIKO I\&E SSC-5000) at a heating rate of $10{ }^{\circ} \mathrm{C} / \mathrm{min}$. Polarized IR spectra were recorded with a JEOL JIR-3505 FT-IR spectrometer with the aid of a gold wire glid polarizer. Oriented samples of the PLC films were prepared in a magnetic field of $2.1 \mathrm{~T}$.

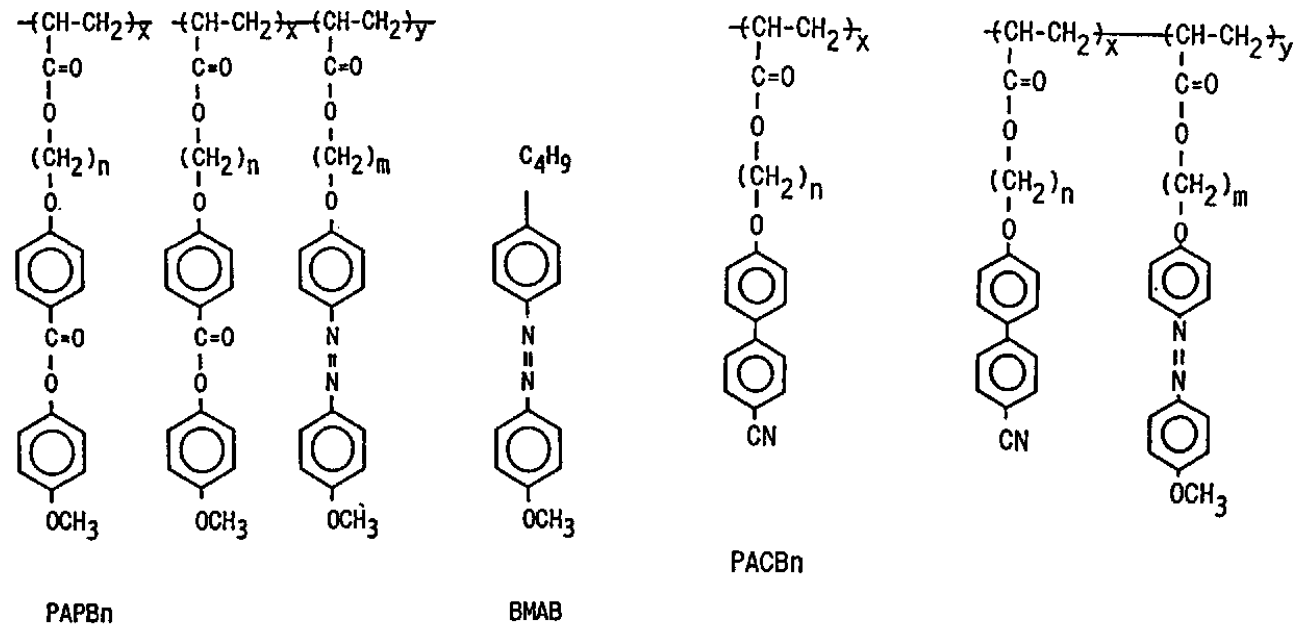

Fig. 1 Structures of PLCs and Photoresponsive Molecules

\section{Photochemical Phase Transition}

Photochemical phase transition behavior of PLCs was followed by monitoring the intensity of the linearly polarized light at $633 \mathrm{~nm}$ from a He-Ne laser transmitted through a pair of crossed polarizers with a photodiode [11]. In this apparatus, the absorbance of the azobenzene derivatives incorporated could be measured in situ at the photoirradiation wavelength. Thus, the photoisomerization behavior of the photochromic molecules was followed simultaneously on photoirradiation. Polymer films were placed in a thermostated block and irradiated with a monochromatic light from a JASCO CRM-FA irradiator.

\section{Results and Discussion}

Thermotropic Properties of PLCs

Fig. 2 shows the phase transition behaviors of the homopolymers (PAPBn and $\mathrm{PACBn}$ ) as a function of $\mathrm{MW}$. $\mathrm{Tg}$ and $\mathrm{Tni}$ (Tsi) of the polymers increased with MW except PACB5, which showed little temperature dependence. 

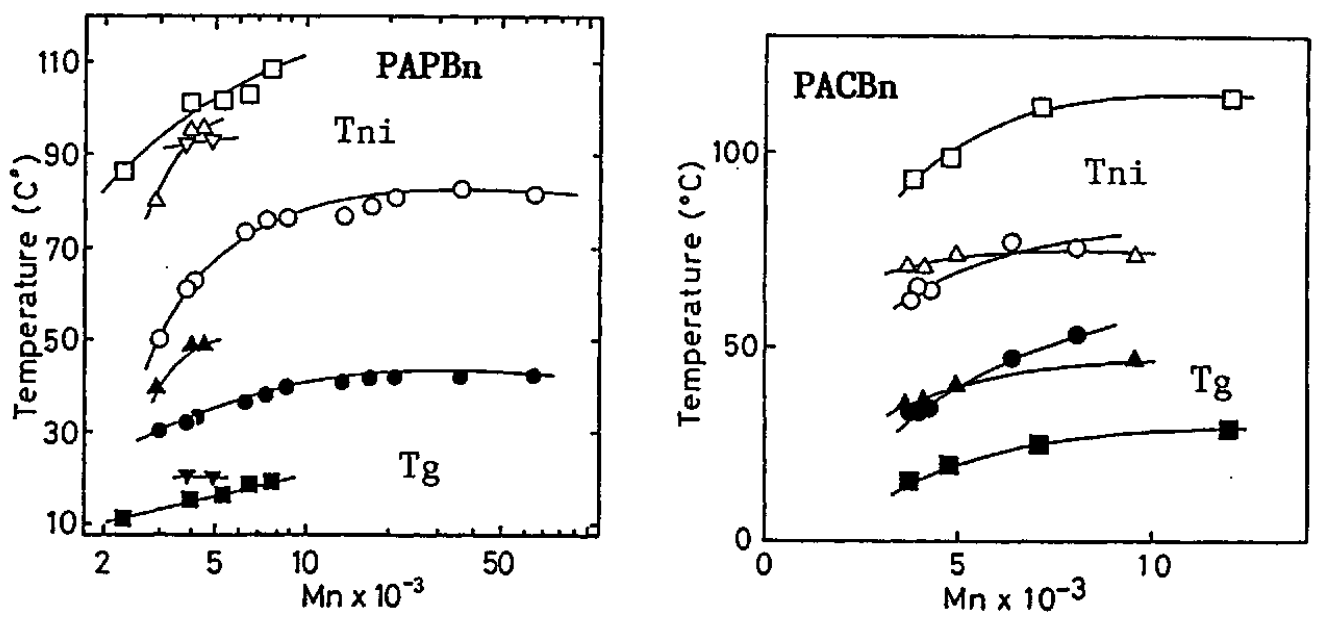

Fig. 2 Phase Transition Temperatures as a Function of Molecular Weight

\section{Order Parameters Determined by IR Dichroism}

Fig. 3 shows the order parameters $(S)$ of PAPBn $(M n=4000)$ and $\mathrm{PACBn}$ $(\mathrm{Mn}=4000)$ as a function of the reduced temperature, which were determined by IR dichroism. Abrupt change in $S$ observed for PAPBn ( $n=5$ and 6) corresponds to the phase transition. It is seen that PAPB3 and PACB3 possess lower values of $S$ than the other polymers approximately in the whole temperature range. Coupled with the extraordinarily low values of the thermodynamic parameters (Tni, $\triangle H$ ni and $\triangle \mathrm{Sni}$ ) of PAPB3 and PACB3, this result clearly indicates that PAPB3 and PACB3 have much less ordered structures in the $N$ state than the other homopolymers. Parallel tendency between Sni and $\mathrm{S}$ can be rationalized if we assume that PLCs have the same values of entropy in the isotropic state. Namely, a large value of Sni means a small value of entropy in the $\mathrm{N}$ state; thus, higher orientational ordering in the $N$ state.
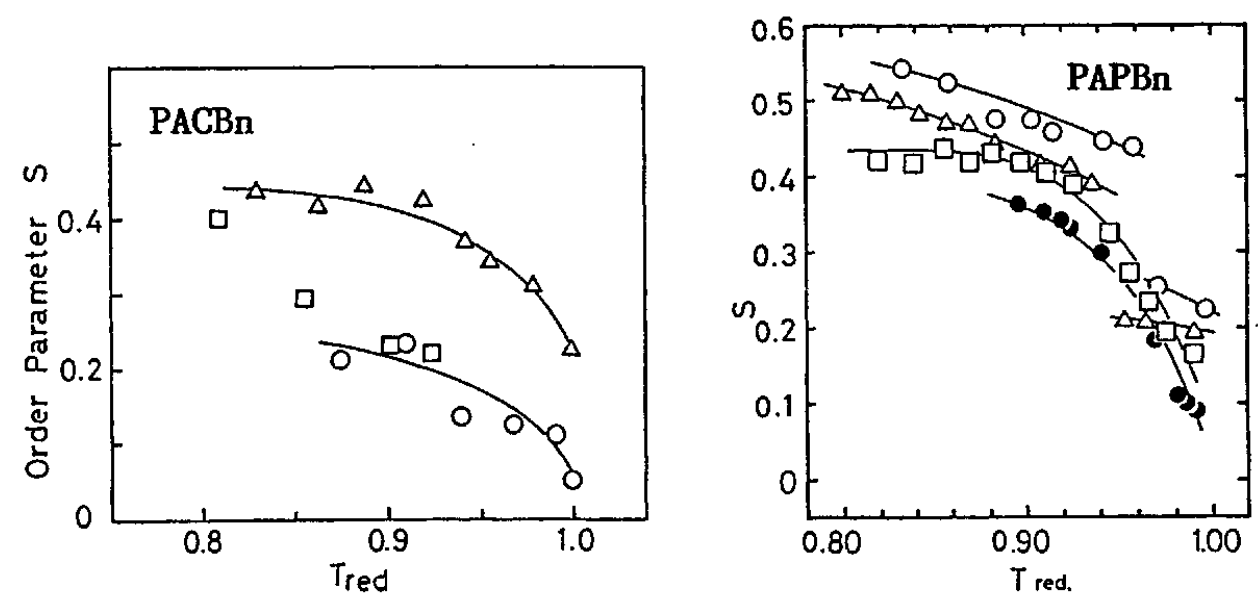

Fig. 3 Order Parameters of PLCs Determined by IR Dichroism. 


\section{Photochemical Phase Transition}

A typical example of the photochemical phase transition behavior is shown in Fig. 4 where a PAPB3 film doped with $5 \mathrm{~mol} \%$ of BMAB was irradiated at $366 \mathrm{~nm}$ or at $525 \mathrm{~nm}$. Transmittance of the linearly polarized light through a pair of crossed polarizers between which the sample was placed, $I(t)$, was measured simultaneously as a function of time. It is clear that trans - cis photoisomerization of the doped BMAB $(366 \mathrm{~nm})$ brought about $\mathrm{N}-\mathrm{I}$ phase transition of the host PLC as demonstrated by complete loss of birefringence $(\mathrm{I}(\mathrm{t})=$ $0)$ and cis - trans isomerization $(525 \mathrm{~nm})$ restored the system to the initial state (N). Reversible phase transition of the PLC can be induced in this way by photochemical reaction of a small amount of the incorporated photoresponsive molecules.

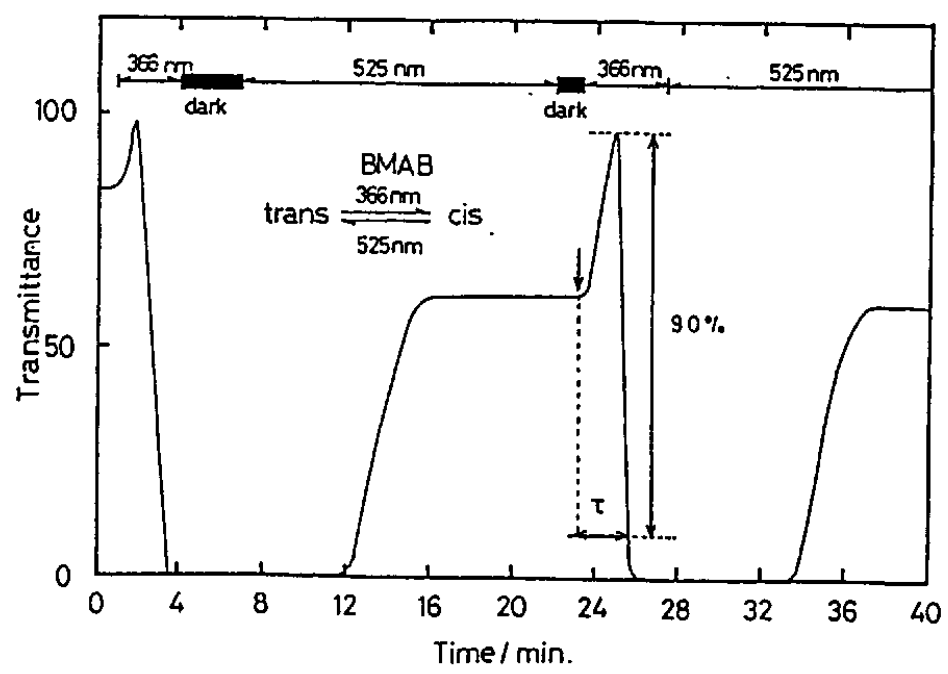

Fig. 4 Photochemical Phase Transition Behaviors of PAPB3 Doped with BMAB. Tred $=0.99 ; \mathrm{BMAB}, 5$ mole $\%$.

It must be mentioned here that long term storage of information can be achieved by cooling the PLC film below $\mathrm{Tg}$ of the polymer. The PAPB3/BMAB film was irradiated at $366 \mathrm{~nm}$ and the $\mathrm{N}$ - I phase transition was confirmed by transmittance of the polarized light. This film was then quickly cooled to $-20^{\circ}$ $\mathrm{C}$ and kept below $\mathrm{Tg}$ of PAPB3 employed $\left(20^{\circ} \mathrm{C}\right)$. Observation of the film with the polarizing microscope revealed that the I phase formed at the site of photoirradiation still remained unchanged after one year.

In order to discuss the phase transition behavior quantitatively, we defined the response time, $\tau$, as the time required to reduce the transmittance across the crossed polarizers, $I(t)$, to $10 \%$ of the maximum value (Fig. 4).

Effect of Molecular Weight and Spacer Length 
In Fig. 5 is shown the molecular weight $(\mathrm{Mn})$ dependence of the photochemical phase transition behavior of PAPBn doped with $5 \mathrm{~mol} \%$ of BMAB. Tni of the homopolymers was dependent both on $\mathrm{Mn}$ and the spacer length (n) as shown in Fig. 2; thus, the photochemical phase transition behavior was examined at Tred $(=\mathrm{T} / \mathrm{Tni})$ of 0.99 for all cases. The rate of the phase transition evaluated by reciprocal of the response time, $\tau_{-1}^{-1}$, was strongly dependent on $\mathrm{Mn}$ of the host PLC. In the case of PAPB3, $\tau^{-1}$ decreased with increasing $\mathrm{Mn}$ and at $\mathrm{Mn}>10^{4}$ no phase transition was induced even after prolonged irradiation. Contrary to the behavior of PAPB3, no phase transition was induced in PAPB2, PAPB5 and PAPB6 on prolonged irradiation even though trans - cis photoisomerization of the doped $\mathrm{BMAB}$ was confirmed by absorption spectroscopy.

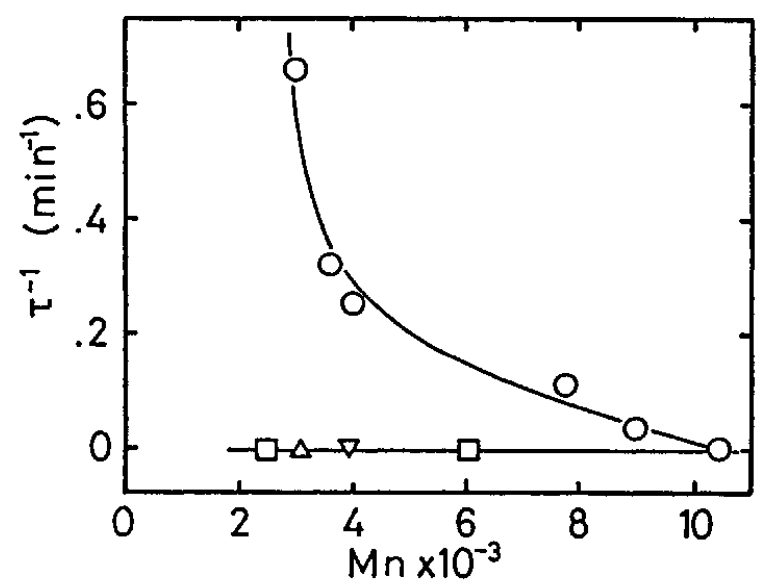

Fig. 5 Effect of $\mathrm{Mn}$ on Photochemical Phase Transition $(\triangle)$, PAPB2; (O), PAPB3; $(\nabla)$, PAPB5; $(\square)$, PAPB6. BMAB, 5 mole\%.

\section{Effect of Temperature}

Fig. 6 shows the temperature dependence of the photochemical phase transition behavior of PAPB3/BMAB (5 mol\%) mixture. One can see that the rate of the phase transition increases with Tred and it is highest at Tred $=0.99$ among the temperatures examined. The rate of the isothermal N - I phase transition is closely related to lowering of the phase transition temperature caused by accumulation of the cis-form of BMAB [13,14]. Namely, Tni decreases as the concentration of the cis-form increases and when Tni of the system is lowered below the irradiation temperature, the isothermal phase transition of the mixture takes place. Thus, when the irradiation temperature is close to Tni of the initial state, the amount of the cis-form required to lower Tni below the irradiation temperature is rather small and the phase transition is induced rapidly. On the other hand, when the irradiation temperature is low, a large amount of 
the cis-form are necessary to lower Tni of the system below the temperature, thus the rate of the phase transition is expected to be low.

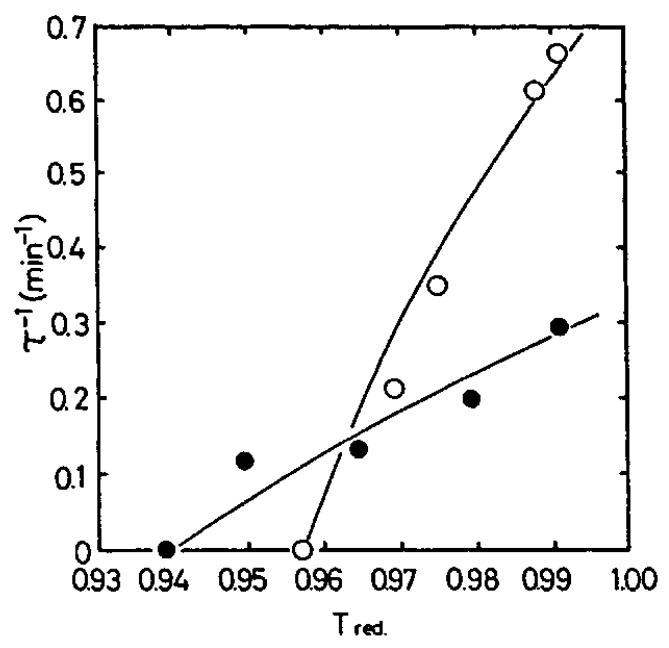

Fig. 6 Temperature Dependence of Photochemical Phase Transition.

(O), $\mathrm{Mn}=3000 ;(\odot), \mathrm{Mn}=3700$.

Comparison of Photochemical Phase Transition Behavior with Thermodynamic Data and Order Parameters

In Fig. 7 is shown the rate of the isothermal $N$ - I phase $\operatorname{transition}\left(\tau^{-1}\right)$ of PLCs as a function of their $\triangle$ S Sni values. Data were taken from the measurements at Tred $=0.99$. All the PLC films that exhibited the photoinduced phase transition are included in the figure. It is evident that $\tau^{-1}$ is well correlated to $\Delta \mathrm{Sni}$, thus the photochemical phase transition takes place effectively in the less ordered system.
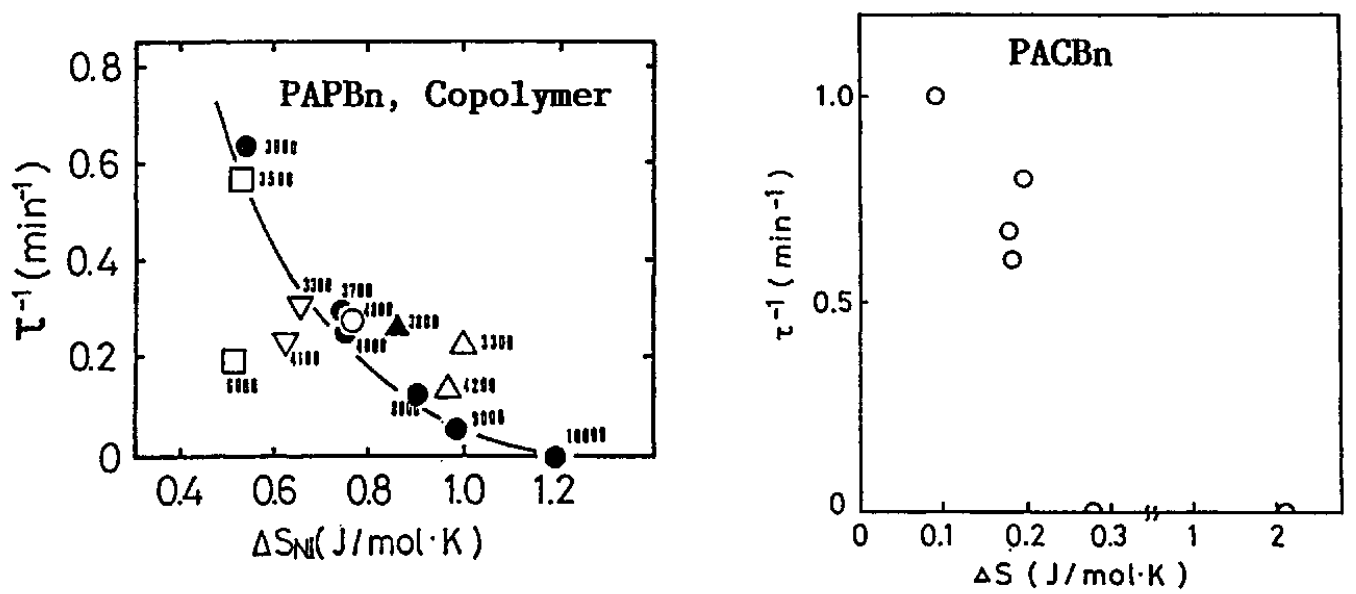

Fig. 7 The Rate of Photochemical Phase Transition vs. Change in Entropy.

Use as Image Storage Materials 
The present study clearly demonstrated that reversible phase transition of the side-chain PLCs could be induced by photochemical reaction of a small amount of the incorporated photoresponsive molecules. From the application point of view, this means that the photosignals are transformed into structural change of the photoreceptor molecules (trans - cis isomerization) at the irradiated sites, inducing the phase change at the irradiated sites. The photosignals transformed into the phase change can be read out by the loss of birefringence. Note that the system is transparent to the monitor light $(633 \mathrm{~nm})$ and consequently, the read-out is completely non-destructive. Merits of this way of image storage are obvious [4]. Since the photosignals are converted to the physical change at the irradiated sites, a variety of methods other than the transmittance of the linearly polarized light described here can be used for the non-destructive read-out of the stored information.

Image storage experiments by the use of a standard photo-mask were conducted on Copolymer PB 3-3 with $\mathrm{Mn}=3500$. The photo-mask was directly placed on the PLC film and the resulting sample was irradiated with the third harmonic $(355 \mathrm{~nm})$ of a Q-switched pulsed Nd:YAG laser (Spectron SL803). Irradiation was performed at $54{ }^{\circ} \mathrm{C}$ (Tred $=0.98$ ). After exposure, the PLC film was cooled to room temperature (below $\mathrm{Tg}$ ) and resolution of the stored image was examined in the polarizing microscope. We obtained a resolution up to 2 um.

\section{References}

1. W. Haas et al., Mol. Cryst. Liq. Crys., 7 (1969) 371.

2. W.E. Haas et al., J. Electrochem. Soc., 121 (1974) 1667.

3. E. Sackmann, J. Am. Chem. Soc., 93 (1971) 7088; K. Ogura et al., Jpn. J. Appl. Phys., 21 (1982) 969;

4. S. Tazuke and T. Ikeda, ACS Symposium Series 381, (1989) p209.

5. T. Yoshizawa, Adv. Biophys., 17 (1984) 5.

6. G. Attard and G. Williams, Nature, 326 (1987) 544.

7. V.P. Shibaev et al., Polym. Commun., 24 (1983) 364.

8. H.J. Coles and R. Simon, Mol. Cryst. Liq. Crys. Lett., 102 (1981) 43; Polymer, 26 (1985) 1801.

9. M. Eich, J.H. Wendorff, Makromol. Chem. Rapid Commun., 8 (1987) 59; 467.

10. S. Tazuke et al., Chem. Lett., 911 (1987); S. Kurihara et al., Jpn. J. Appl. Phys., 27 (1988) L1791; Mol. Cryst. Liq. Cryst., 178 (1990) 117; T. Ikeda et al., J. Phys. Chem., 95 (1990) 6550.

11. T.Ikeda et al., Chem. Lett., 1679 (1988); Macromolecules, 23 (1990) 36; 23 (1990) 42; 23 (1990) 3938; 23 (1990) 1691; Mol. Cryst. Liq. Cryst., 182B (1990) 357; 182B (1990) 373; 188 (1990) 207; 188 (1990) 223; 188 (1990) 235. 